\title{
El odontopediatra del futuro para tiempos post COVID-19.
}

\section{The pediatric dentist of the future for post COVID-19 times.}

\author{
Vidal Pérez ${ }^{1 *}$, Jaime Díaz ${ }^{2}$, Sandra Rojas ${ }^{3}$, Juan Eduardo Onetto ${ }^{4}$
}

1. Departamento de Pediatría Estomatológica, Facultad de Ciencias de la Salud, Universidad de Talca. Chile.

2. Departamento de Odontopediatría y Ortodoncia, Facultad de Odontología, Universidad de La Frontera. Chile.

3. Departamento del Niño y Ortopedia

Dentomaxilar, Facultad de Odontología, Universidad de Chile. Chile.

4. Cátedra de Odontopediatría, Facultad de Odontología, Universidad de Valparaíso. Chile.

*Correspondencia a: Vidal Pérez. | Universidad de Talca. Av. Lircay s/n, Talca, Chile. | Teléfono: +56 712203026 (extensión 3026) | E-mail: vperez@ utalca.cl

Trabajo recibido 03/06/2020

Aprobado para su publicación 08/06/2020
Desde la llegada del coronavirus SARS-CoV-2 a nuestro país en marzo 2020, la enfermedad COVID-19 se ha diseminado entre nuestra población provocando, como en todo el mundo, cambios significativos en nuestros estilos de vida ${ }^{(1,2)}$. Esta pandemia ha golpeado a la odontología y la odontopediatría en particular en sus ámbitos profesionales y formativos ${ }^{(3,4)}$. A pesar de nuestros esfuerzos, el retorno a la actividad clínica requerirá la adquisición de nuevas competencias transversales importantes en la formación del odontopediatra que requerirá nuestra sociedad en los tiempos post COVID-19(5,6).

Luego del arribo de este virus, las universidades chilenas comenzaron a utilizar masivamente plataformas virtuales y recursos digitales, no exento de dificultades ${ }^{(7)}$. Las escuelas y facultades de odontología siguieron esos lineamientos con un ajustado plan de trabajo para enfrentar los desafíos que involucra la enseñanza de la odontología y particularmente de la odontopediatría en tiempos de COVID-19. Durante este primer semestre, los programas curriculares de pre y postgrado han sido adaptados para una docencia teórica online, al igual que en todo el ámbito de la educación superior ${ }^{(8)}$. Por otro lado, las actividades clínicas han sido suspendidas en forma temporal, a la espera de la resolución sanitaria del Ministerio de Salud que permita volver con las normas de bioseguridad adecuadas a las actividades académicas en los diferentes campos clínicos. Aunque todas las instancias universitarias promueven una búsqueda de medidas que protejan a profesores, estudiantes (residentes), personal administrativo y pacientes, se ve incierto el retorno a la actividad clínica en el segundo semestre.

Los académicos hemos aplicado una variedad de estrategias metodológicas para entregar los contenidos teóricos, de manera de lograr los objetivos de aprendizaje planificados. Entre éstas se destacan: clases asincrónicas y sincrónicas, talleres de resolución de problemas, foros para resolver dudas y canales de YouTube para almacenar las creaciones docentes de libre acceso para los estudiantes ${ }^{(5,7,8)}$. Paralelamente, se han diseñado una serie de evaluaciones formativas que refuerzan los contenidos presentados en clases y talleres. Todo lo anterior, tratando de respetar los tiempos de trabajo autónomo y brindando la contención emocional que promueva la resiliencia necesaria (que amerita el contexto social que estamos viviendo), ya que, tanto estudiantes como académicos estamos en un proceso de aprendizaje digital continuo ${ }^{(9,10)}$. Sin embargo, estamos ciertos que la enfermedad COVID-19 impactará significativamente la forma de enseñar odontología y especialmente odontopediatría en el futuro inmediato ${ }^{(11-13)}$. Al respecto, la restricción de la atención clínica que ha establecido la Autoridad Sanitaria en Chile, que autoriza solo atenciones de urgencias, nos obligan a analizar profundamente la forma en que regresaremos a la atención clínica de pacientes, tanto en el contexto público, privado y en la academia.

Posiblemente y de acuerdo a lo observado en múltiples publicaciones e informes de diferentes instancias rectoras de salud en el mundo, realizar un diagnóstico a distancia de las condiciones de salud general y bucal del paciente a través de tele-odontología, control de temperatura, desinfección de calzado y lavado frecuente de manos, entre otras, serán procedimientos habituales en nuestra práctica clínica futura ${ }^{(14-16)}$. Una adecuada desinfección de hall de ingresos, salas de espera y box clínicos junto con el uso y recambio de los equipos de protección personal, serán procedimientos de rutina que extenderán los tiempos en el tratamiento de pacientes, reduciendo el número de atenciones ${ }^{(17,18)}$. En este escenario complejo el desarrollo de nuevas tecnologías (como sistemas de aspiración, nuevos agentes de irrigación, entre otros) que reduzcan la carga vírica de los ambientes de trabajo serán necesarias ${ }^{(19,20)}$. Sin embargo, estos aspectos son solo una parte de los cambios que requerirá nuestra profesión y particularmente nuestra especialidad.

Es probable que esta pandemia despierte en la odontología en general y odontopediatría en particular, la necesidad de métodos alternativos de entrenamiento que permitan a nuestros especialistas el desarrollo de competencias acordes a los tiempos post COVID-19 que viviremos $^{(5,11)}$. Entonces, es de la mayor importancia que las instituciones de educación superior, sus escuelas y facultades de odontología actúen en forma rápida y proactiva reevaluando el perfil de egreso de sus estudiantes pre y postgrado. Por lo anterior, se hace necesario entonces repensar las competencias que requiere este "nuevo odontopediatra".

El odontopediatra del futuro debería ser hábil en el uso de las Tecnologías de la Información y Comunicación (TIC) a distancia existentes y emergentes, por lo que las habilidades telemáticas (propias de la ingeniería) son saberes a incorporar en su plan de estudio(21,22). Tan importante como lo anterior, las habilidades comunicativas de nuestro especialista serán altamente requeridas para transmitir los conceptos de promoción y prevención en salud bucal tanto a nivel comunitario como individual(23-25). En este contexto, la educación de los pacientes y sus familias requerirá un dentista hábil en observar la "realidad del paciente y su entorno biopsicosocial" y utilizar esa información como un instrumento que facilite la educación en salud bucal. Por lo anterior, se hace necesario considerar decididamente el uso de estrategias educativas basadas en los conceptos de la entrevista motivacional u otras similares, siendo alternativas a implementar 
en los programas de especialización en odontopediatría(26-28).

Toda competencia tiene una parte procedimental, pero ¿cómo evaluar si un estudiante es competente para realizar un procedimiento odontológico? ¿cuántas acciones o procedimientos debe realizar el estudiante para demostrar que adquirió una determinada competencia en odontopediatría? Estas son preguntas difíciles de responder y posiblemente se debe a que la formación histórica y tradicional del odontólogo, se sustenta principalmente en la adquisición de habilidades a través de la realización de un número repetitivo de técnicas y procedimientos clínicos. Este escenario, sin embargo, no lo tendremos en los tiempos post COVID-19 y es recomendable reconocer que la cantidad de pacientes que atenderán nuestros estudiantes de postgrado será significativamente menor a lo atendido en años anteriores.

En vista de lo anterior, la academia deberá realizar profundas modificaciones en el plan de estudio de los programas de especialización en odontopediatría. Vemos con optimismo que nuevas alternativas de enseñanza junto con el establecimiento de los conceptos y fundamentos de odontología de mínima intervención, serán la base del desarrollo de la odontopediatría del futuro ${ }^{(29,30)}$. Este tipo de acercamiento permitirá entregar al paciente ( $\mathrm{y}$ a su grupo familiar) las herramientas necesarias para hacerlo responsable de su propia salud bucal de manera informada. Este acercamiento también incluye, el uso racional de fluoruros supeditado a un diagnóstico de riesgo cariogénico previo. Al momento de intervenir, se realizarán las alternativas terapéuticas que sean menos invasivas para el paciente; por lo que conceptos como la eliminación selectiva de lesiones de caries y el uso de cementos de vidrio ionómero permitirá reducir la posibilidad de terapias pulpares y proteger al máximo el remanente dentario(30-32). Por último, el tratamiento no invasivo de lesiones de caries interproximales, as como el uso de coronas metálicas (técnica de Hall) y terapias pulpares con pastas antibióticas son procedimientos rehabilitadores que deberían ser parte del plan de estudio de nuestro odontopediatra del futuro(33-36).

El propósito de este artículo, es hacer un llamado a repensar las adecuaciones indispensables que nuestra especialidad demanda con urgencia y reflexionar acerca del odontopediatra que requiere nuestra sociedad para los tiempos post pandemia. Los nuevos odontopediatras serán odontólogos integrales, líderes en odontología de mínima intervención, tendrán competencias en tecnologías de la información y comunicación que les permitirán hacer de la promoción/prevención la base del autocuidado en salud bucal de su paciente y grupo familiar. Para esto, debemos contribuir con los cambios curriculares adecuados que permitan esa formación.

Entre los cambios mencionados consideramos relevante:

1) Favorecer el desarrollo de habilidades blandas, comunicación oral, escrita y trabajo en equipo, en nuestros estudiantes de postgrado.

2) Incorporar los conceptos de entrevista motivacional para abordar los cambios de conducta en salud bucal.

3) Incorporar competencias de teleodontología relacionadas con el uso de las TIC, con un enfoque integral.

4) Promover el autocuidado en salud oral a nivel individual y comunitario.

5) Realzar la filosofía y aplicación de los conceptos de odontología de mínima intervención, como el centro de la formación del futuro odontopediatra.

6) Desarrollar la ética del conocimiento, como la base en la toma de decisiones clínicas.

Ha pasado el tiempo de observar y adaptarnos a la realidad que nos tocó vivir con el COVID-19. Ahora es tiempo de dialogar, crear y hacer que las cosas pasen. La odontopediatría del futuro nos puso aquí para eso.

\section{CONFLICTO DE INTERÉS}

Los autores declaran no presentar conflicto de intereses.

\section{Bibliografía}

1. Gobierno de Chile. Plan de acción CORONAVIRUS COVID-19 [Internet]. Santiago: Gobierno de Chile; 2020. [consultado 28/05/2020]. Disponible en: https://www.gob.cl/ coronavirus/cifrasoficiales/.

2. WHO. Coronavirus disease (COVID-19) Situation Report - 124 Geneva: World Health Organization; 2020. [consultado 28/05/2020]. Disponible en: https://www.who. int/docs/default-source/coronaviruse/situation-reports/20200523-covid-19-sitrep-124 pdf?sfvrsn=9626d639 2.

3. Singh V, Lehl GK, Talwar M, Luthra A. The novel coronavirus and challenges for general and paediatric dentists. Occup Med (Lond). 2020. May 2;. doi: 10.1093/occmed/ kqaa055. Online ahead of print

4. Flores M, Onetto J, Jamett J, Sievers D. Odontología en tiempos de pandemia: desafíos para una nueva época 2020 [consultado 31/05/2020]. Disponible en: https:// facultadodontologia.uv.cl/images/Documentos/odontologiaenpandemia.pdf.

5. Quinn B, Field J, Gorter R, Akota I, Manzanares MC, Paganelli C, et al. COVID-19: The immediate response of European academic dental institutions and future implications for dental education. Eur J Dent Educ. 2020 May 11; doi: 10.1111/eje.12542.Online ahead of print.

6. Iyer P, Aziz K, Ojcius DM. Impact of COVID-19 on dental education in the United States. J Dent Educ. 2020 Apr 27. doi: 10.1002/jdd.12163.Online ahead of print.

7. MINEDUC. Apoyos del Mineduc durante la pandemia del COVID-19 Santiago: Ministerio de Educación; 2020. [consultado 28/05/2020]. Disponible en: https://www.mineduc.cl/apoyos-del-mineduc-durante-la-pandemia-del-covid-19/.

8. Consejo de Rectores de las Universidades Chilenas. Comunicado público Santiago: Consejo de Rectores de las Universidades Chilenas; 2020. [consultado 28/052020] Disponible en: https://www.consejoderectores.cl/noticia/865/universidades-del-consejode-rectores-suspenden-clases-para-prevenir-contagio-de-coronavirus.html.

9. Zhai Y, Du X. Addressing collegiate mental health amid COVID-19 pandemic. Psychiatry Res. 2020;288:113003.

10. Montemurro N. The emotional impact of COVID-19: From medical staff to common people. Brain Behav Immun. 2020. Mar 30. doi: 10.1016/j.bbi.2020.03.032. Online ahead of print

11. Dragan IF, Walji M, Vervoorn M, Quinn B, Johnson L, Davis J, et al. ADEA-ADEE shaping the future of dental education III: The impact of scientific technologies and discoveries on oral health globally. J Dent Educ. 2020;84(1):111-6.

12. Mariño R, Ramos-Gómez F, Manton DJ, Onetto JE, Hugo F, Feldens CA, et al. The future of pediatric dentistry education and curricula: a Chilean perspective. BMC Oral Health. 2016 Jul 18;17(1):20. doi: 10.1186/s12903-016-0251-

13. Casamassimo PS, Townsend JA, Litch CS. Pediatric dentistry during and after COVID-19. Pediatr Dent. 2020;42(2):87-90.

14. AAPD. A guide for re-entry into practice for pediatric dentist during the COVID-19 pandemic Chicago: American Acamedy of Pediatric Dentistry; 2020 [consultado 28/05/2020]. Disponible en: https://www.aapd.org/globalassets/media/covid-19/aapdpracticechecklist.pdf.

15. Bradley M, Black P, Noble S, Thompson R, Lamey PJ. Application of teledentistry in oral medicine in a community dental service, N. Ireland. Br Dent J. 2010;209(8):399 404

16. Maret $D$, Peters $O A$, Vaysse $F$, Vigarios $E$. Integration of telemedicine into the public health response to COVID-19 must include dentists. Int Endod J. 2020;53(6):880-1. 17. Ather A, Patel B, Ruparel NB, Diogenes A, Hargreaves KM. Coronavirus Disease 19 (COVID-19): Implications for clinical dental care. J Endod. 2020;46(5):584-95.
18. DIPRECE. Orientaciones para atención odontológica en fase IV COVID-19 Santiago: División de Prevención y Control de Enfermedades; 2020 [consultado 28/05/2020] Disponible en: https://diprece.minsal.cl/wp-content/uploads/2020/03/ORIENTACIONES-ATENCION-ODONTOLOGICAS-COVID-19-.pdf.

19. Jacks ME. A laboratory comparison of evacuation devices on aerosol reduction. J Dent Hyg. 2002;76(3):202-6.

20. Harrel SK, Molinari J. Aerosols and splatter in dentistry: a brief review of the literature and infection control implications. J Am Dent Assoc. 2004;135(4):429-37.

21. Smith AC, Thomas E, Snoswell CL, Haydon H, Mehrotra A, Clemensen J, et al. Telehealth for global emergencies: Implications for coronavirus disease 2019 (COVID-19). J Telemed Telecare. 2020:1357633X20916567.

22. Machado RA, de Souza NL, Oliveira RM, Martelli Júnior H, Bonan PRF. Social media and telemedicine for oral diagnosis and counselling in the COVID-19 era. Oral Oncol. 2020;105:104685.

23. Freeman R. Communicating with children and parents: recommendations for a childparent-centred approach for paediatric dentistry. Eur Arch Paediatr Dent. 2008;9 Suppl 1:16-22.

24. Alvarez S, Schultz JH. A communication-focused curriculum for dental students - an experiential training approach. BMC Med Educ. 2018;18(1):55.

25. Field J, Stone S, Orsini C, Hussain A, Vital S, Crothers A, et al. Curriculum content and assessment of pre-clinical dental skills: A survey of undergraduate dental education in Europe. Eur J Dent Educ. 2018;22(2):122-7.

26. Gillam DG, Yusuf H. Brief motivational interviewing in dental practice. Dent J (Basel) 2019 May 1;7(2):51.

27. Lundahl B, Moleni T, Burke BL, Butters R, Tollefson D, Butler C, et al. Motivational interviewing in medical care settings: a systematic review and meta-analysis of randomized controlled trials. Patient Educ Couns. 2013;93(2):157-68.

28. Wu L, Gao X, Lo ECM, Ho SMY, McGrath C, Wong MCM. motivational interviewing to promote oral health in adolescents. J Adolesc Health. 2017;61(3):378-84

29. Lugassy D, Levanon Y, Shpack N, Levartovsky S, Pilo R, Brosh T. An interventional study for improving the manual dexterity of dentistry students. PLoS One. 2019;14(2):e0211639.

30. Banerjee A. 'Minimum intervention' - Ml inspiring future oral healthcare? Br Dent J. 2017;223(3):133-5.

31. Innes NP, Frencken JE, Bjørndal L, Maltz M, Manton DJ, Ricketts D, et al. Managing carious lesions: Consensus recommendations on terminology. Adv Dent Res. 2016;28(2):49-57

32. Toumba KJ, Twetman S, Splieth C, Parnell C, van Loveren C, Lygidakis N. Guidelines on the use of fluoride for caries prevention in children: an updated EAPD policy document. Eur Arch Paediatr Dent. 2019;20(6):507-16

33. Amaechi BT. Remineralisation - the buzzword for early MI caries management. $\mathrm{Br}$ Dent J. 2017;223(3):173-82.

34. Innes NP, Evans DJ, Bonifacio CC, Geneser M, Hesse D, Heimer M, et al. The Hall Technique 10 years on: Questions and answers. Br Dent J. 2017;222(6):478-83.

35. de Deus Moura LeF, de Lima MeD, Lima CC, Machado JI, de Moura MS, de Carvalho PV. Endodontic Treatment of primary molars with antibiotic paste: A Report of 38 Cases. J Clin Pediatr Dent. 2016;40(3):175-7.

36. Reddy GA, Sridevi E, Sai Sankar AJ, Pranitha K, Pratap Gowd MJS, Vinay C. Endodontic treatment of chronically infected primary teeth using triple antibiotic paste: An. J Conserv Dent. 2017;20(6):405-10. 